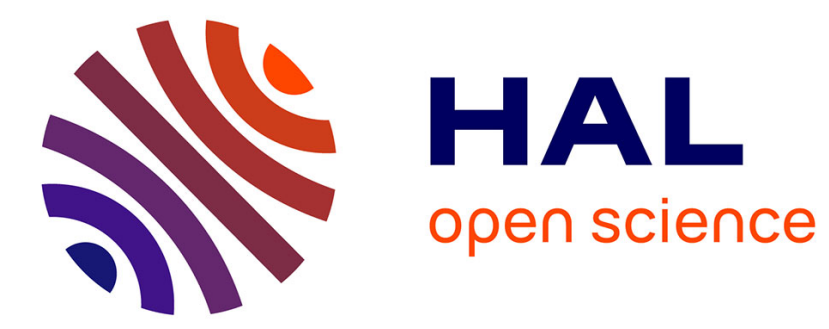

\title{
Douceurs somatiques
}

Isabelle Ginot

\section{To cite this version:}

Isabelle Ginot. Douceurs somatiques. Repères, cahier de danse, 2013, 10.3917/reper.032.0021 . hal02495628

\section{HAL Id: hal-02495628 \\ https://hal.science/hal-02495628}

Submitted on 12 Apr 2020

HAL is a multi-disciplinary open access archive for the deposit and dissemination of scientific research documents, whether they are published or not. The documents may come from teaching and research institutions in France or abroad, or from public or private research centers.
L'archive ouverte pluridisciplinaire HAL, est destinée au dépôt et à la diffusion de documents scientifiques de niveau recherche, publiés ou non, émanant des établissements d'enseignement et de recherche français ou étrangers, des laboratoires publics ou privés. 


\section{DOUCEURS SOMATIQUES}

Isabelle Ginot

La Briqueterie / CDC du Val-de-Marne | «Repères, cahier de danse »

2013/2 n³2 | pages 21 à 25

ISSN 2112-5147

Article disponible en ligne à l'adresse :

https://www.cairn.info/revue-reperes-cahier-de-danse-2013-2-page-21.htm

\section{Pour citer cet article :}

Isabelle Ginot, «Douceurs somatiques », Repères, cahier de danse 2013/2 (n³2), p. 21-25.

DOI 10.3917/reper.032.0021

Distribution électronique Cairn.info pour La Briqueterie / CDC du Val-de-Marne.

(c) La Briqueterie / CDC du Val-de-Marne. Tous droits réservés pour tous pays.

La reproduction ou représentation de cet article, notamment par photocopie, n'est autorisée que dans les limites des conditions générales d'utilisation du site ou, le cas échéant, des conditions générales de la licence souscrite par votre établissement. Toute autre reproduction ou représentation, en tout ou partie, sous quelque forme et de quelque manière que ce soit, est interdite sauf accord préalable et écrit de l'éditeur, en dehors des cas prévus par la législation en vigueur en France. Il est précisé que son stockage dans une base de données est également interdit. 


\title{
Jlouceurs somatiçıes
}

\author{
par Isabelle Ginot \\ En quoi les « méthodes douces » sont-elles douces? \\ Quelles valeurs - et quelles normes - sous-tendent \\ ces pratiques, qui irriguent largement le champ \\ de la danse aujourd'hui?
}

ISABELLE GINOT

est enseignante au département Danse de l'université Paris 8 et praticienne Feldenkrais. Après une période de travail, recherche et publications sur les œuvres en danse contemporaine et les pratiques qui les accompagnent (analyse des œuvres, analyse des pratiques et des processus), elle s'est tournée vers un champ particulièrement mal connu, celui des pratiques somatiques, pratiques somatiques, de leur histoire, de leu épistémologie et de leurs usages auprès des publics dits «fragiles». Elle est responsable du DU « Techniques du corps et monde du soin" (Paris 8/ service Formation permanente).

La plupart de ses publications son disponibles sur le site internet Paris 8 Danse.

NOTE

1. J'emploie ici ce terme dans son sens géologique initial, à savoir, «doctrine géologique établie par Stanislas Meunier, et montrant dans toute son étendue l'activité chimique des masses minérales » (Centre National de Ressources Textuelles et Lexicales, page consultée le 9 octobre 2013). Autrement dit, pour décrire l'intensité de l'activité perceptive invisible, cause et source de "l'état actuel » du geste visible. Sans exclure pour autant, bien au contraire, la signification d'activite politique intense.
Nombreux sont les danseurs qui, parallèlement à leur entraînement "traditionnel ", ont recours à des pratiques corporelles qu'on appelle «douces", ce terme imprécis incluant les pratiques "somatiques", mais aussi parfois les techniques de danse "release» et parfois même les arts martiaux - notamment les arts martiaux "intérieurs " comme le Qi Gong ou le Tai Chi, qui n'incluent pas de techniques de combat. L'expression "méthodes douces » fait écho à celle de "médecines douces», et souligne l'ambivalence des Somatiques quant à la question thérapeutique, certaines méthodes se revendiquant comme thérapeutiques alors que d'autres préfèrent se définir comme éducatives. Si ce terme de "douceur» n'est guère défini ou justifié, il circule dans les représentations sur ces méthodes, entretenant l'idée que, comme pour la médecine, il y aurait des techniques corporelles «douces» qui s'opposeraient à d'autres, «non douces». Les méthodes somatiques sont-elles douces, et si oui, de quelles douceurs s'agit-il? Et sont-elles «seulement douces» et «toujours douces»? Face à ces questions, je tenterai d'abord de décrire les douceurs somatiques dans leur diversité. Puis, j’affronterai la question, aussi, des violences possibles des somatiques et des risques qu'elles impliquent.

\section{BASSES INTENSITÉS}

Un premier registre de «douceurs » relève de l'évidence: les Somatiques cultivent de basses intensités; la lenteur, par opposition aux vitesses extrêmes recherchées dans de nombreuses pratiques corporelles, qu'elles soient dansées ou sportives; la réduction de l'effort et le primat donné à l'écoute des sensations fines, par opposition à l'exploit physique, intense, et aux sensations dites de l'extrême; l'exploration des prémices du mouvement, et non la recherche de son aboutissement; la recherche du mouvement sans blessure, par opposition à la recherche de l'exploit au risque des blessures. Ces basses intensités forment une douceur par différence avec la virulence du traitement des corps partout où dominent sensations et efforts extrêmes, valorisation de la douleur et de l'exploit, orientation vers le résultat plus que vers le processus. Elles sont perçues comme douces non seulement par les danseurs, qui y trouvent une alternative aux en- traînements traditionnels, mais aussi par les usagers de la médecine - ceux du moins qui ont l'occasion de les rencontrer -, du sport (de l'amateur au haut niveau), ou encore tous ceux qui perçoivent leur quotidien comme «violent» (stress, cadences, gestes répétitifs, etc.), tant il est vrai que les valeurs qui touchent au geste et au corps, dans toutes les sphères sociales, demeurent du côté de l'extrême et de l'excès. Ces méthodes seraient donc d'abord «douces au corps», parce qu'elles n'exposeraient pas, ou moins, au risque de blessure; comme les «médecines douces» elles seraient non toxiques, elles garantiraient la prévention et la réparation des blessures gagnées dans des pratiques «non douces». Leurs premières douceurs seraient donc celles de leurs qualités de geste: lenteur, petite amplitude, faible intensité, relâchement, travail imaginaire ou visualisation... qualités qui font parfois confondre «douceur» avec passivité, mollesse, voire paresse.

\section{DOUCEURS ACTIVES}

Pourtant, cette douceur que certains associent à de la passivité est indissociable d'une "activité », voire d'un "activisme ${ }^{1}$ » qui échappe souvent aux représentations courantes sur ces méthodes. En effet, les pratiques somatiques ne se basent pas seulement sur une approche du corps et du geste qui les rapproche du champ des médecines "complémentaires", mais aussi sur une pensée de l'apprentissage qui fait écho avec les courants «alternatifs » de la pédagogie, qui se regroupent sous le terme de «méthodes actives ${ }^{2} »$. Plus encore, leurs basses intensités sont la condition de cet "activisme» trop souvent passé sous silence, alors qu'il constitue le cœur de ces pratiques, et qu'il les différencie radicalement de toutes les autres pratiques du corps et du geste.

En premier lieu, un activisme perceptif. En contrepartie et «à condition» du tonus paisible, des amplitudes modérées et des dynamiques tranquilles, les Somatiques exigent une attention extrême, à la fois férocement précise ("savez-vous quelle est la direction de la pointe de votre omoplate lorsque vous faites tel mouvement?»), et infiniment étendue ("pouvez-vous continuer à sentir votre omoplate, et inclure dans votre attention vos contacts avec le sol, la conscience de vos partenaires, > 
> l'espace autour de vous et au-delà...?»). On pourrait finalement décrire les Somatiques comme une discipline d'érudition du sentir. Dans les diverses méthodes somatiques, cette érudition invisible porte des appellations variées (conscience, prise de conscience, attention) qui font écho, dans l'approche des pédagogies actives, à la participation active de l'élève à son propre apprentissage, notamment avec tous les dispositifs d'auto-évaluation et connaissance de sa position dans un programme d'apprentissage.

C'est cet activisme perceptif qui fait des pédagogies somatiques des méthodes actives au sens où on l'entend en pédagogie: celles-ci sont centrées sur l'activité de l'élève; l'enseignant ne délivre pas un savoir à des élèves supposés passifs, mais organise des dispositifs permettant à ceux-ci de découvrir et construire ces savoirs. Il s'agit de mettre en lumière les processus de la connaissance plutôt que les savoirs constitués; les compétences à mobiliser en premier lieu sont celles de l'observation, du non-jugement, de la non-directivité, de l'écoute active, etc. Parmi les notions-clés, citons encore la «situation-problème», qui décrit de près la pédagogie spécifique des cours collectifs de Feldenkrais: pour être résolue, la situation-problème demande à l'élève de mobiliser des savoirs déjà disponibles, mais induit des recherches, des associations, des relations avec le contexte qui font émerger des savoirs nouveaux (en Feldenkrais, de nouvelles coordinations). En pratique corporelle, la pédagogie "problème » s'oppose à la pédagogie du modèle. L'apprentissage concerne donc moins l'acquisition d'une virtuosité formelle que l'élargissement d'un potentiel de geste ${ }^{3}$, autrement dit l'acquisition des conditions favorables à toute virtuosité.

Les Somatiques, depuis le début du XXe , ont développé un spectre large d'éviction de la démonstration: travail les yeux fermés, exploration libre, instructions verbales, nombreuses variations autour de ce qu'en danse on appelle improvisation, multiples formes de "partitions »... Elles sont donc «actives» parce qu'elles proposent moins des modèles à reproduire que des conditions pour inventer son propre geste. Aussi, elles ne s'appuient pas sur une batterie de normes d'évaluation des résultats, mais sur une batterie de ruses conduisant chacun à se situer dans son propre apprentissage. Il ne s'agit pas de se " comparer » aux autres, ni à un modèle à atteindre (dont on serait plus ou moins proche), mais de percevoir changements, différences, éventuellement améliorations, et ainsi, d'établir une échelle de valeurs basée sur sa propre expérience.

Les pédagogies actives se sont constituées comme alternatives à des pédagogies plus traditionnelles, mais aussi comme critiques de l'idéologie sous-jacente à celles-ci. Les méthodes actives présupposent l'égalité des apprenants (et leur droit égal à l'apprentissage), elles s'efforcent de penser aussi l'égalité entre apprenants et enseignants, et, enfin, elles visent à la formation d'adultes (ou futurs adultes) autonomes, libres, plus qu'à la production de sujets soumis aux normes sociales: elles s'opposent radicalement à une logique de formation disciplinaire. On comprend que la critique qu'elles adressent à l'éducation dominante, et tout particulièrement au monde de l'école, soit entendue par le monde de la danse, où dominent les traditions de discipline, d'assujettissement tant à l'autorité du maître, du chorégraphe, que de la forme et de l'instrumentalisation du corps. 


\section{Quand les basses intensités deviennent la norme à atteindre, elles font des Somatiques une morale}

\author{
NOTES \\ 2. La notion de \\ "méthodes actives" \\ ou "pédagogie active " \\ s'emploie couramment \\ pour l'ensemble des \\ pédagogies dites \\ aussi «nouvelles \\ pédagogies » qui \\ traversent tout le \\ vingtième siècle, et \\ qui sont égalemen \\ liées à l'histoire de \\ la psychologie et \\ de la psychanalyse \\ parmi les grands \\ pédagogues de ces \\ courants: Montessori, \\ Freinet, Ferrière, \\ Oury, Decroly... \\ Voir par exemple le \\ Voir par exemple \\ ite des très actifs \\ CEMEA ( Centres \\ d'entraînement aux \\ méthodes d'éducation \\ active»), fondés \\ en 1937, et qui par \\ ailleurs ont été un \\ soutien important \\ de la diffusion de
} l'Eutonie en France: www.cemea.asso.fr/ À ma connaissance, aucune recherche ne s'est encore intéressée aux entrelacements du développement des Somatiques et des pédagogies dites actives. Pourtant, elles partagent des moments historiques communs (par exemple les année 1920-30 en Allemagne, les années 1960-70 en Europe et aux États Unis), it au moins que certains des fondateurs somatiques ont été proches de fondateurs pédagogues importants.

3. Hubert Godard développe cette notion de "potentiel » notamment dans Des trous noirs, entretien avec Patricia Kuypers, in Nouvelles de Danse, $n^{\circ} 53$, Scientifiquement Danse, 2006, p. 56-75.

4. Voir les "chorégraphies de l'attention » dans les pièces de Myriam Gourfink, par exemple.

5. J'ai développé ailleurs cette critique de la «soma-esthétique» de Richard Shusterman: «Discours,

techniques du corps et technocorps", in P. Gioffredi (dir.) A l la'r]encontre de la danse contemporaine: porosités et résistances, L'Harmattan, 2009.
De fait, tandis que l'éducateur somatique se retire dans l'écoute et la non-directivité, et surtout le non-jugement (ce dernier étant particulièrement sensible pour les danseurs), la pédagogie somatique exige des élèves ou participants de constantes prises de décision, explorations autonomes ou à plusieurs, inventions de gestes nouveaux et évaluations des changements. Elle construit, dans le registre du geste, les conditions de l'autonomie, de l'auto-construction des valeurs du sentir. Enfin, elle ne préjuge pas des "résultats", puisqu'elle laisse à chacun l'élaboration de son propre projet au sein de la séance ou d'un ensemble de séances. Ainsi, l'activisme perceptif est profondément attaché aux valeurs de l'action, de l'autonomie et de la mise à distance des normes gestuelles. Souvent «réduites » dans le monde de la danse à une fonction sanitaire (prévenir et réparer les blessures), les Somatiques et leurs basses intensités y ont donc aussi tenu une fonction radicalement politique de critique de ses normes hiérarchiques, pédagogiques et esthétiques.

\section{POLITIQUES DE LA DOUCEUR}

De fait, depuis les années quatre-vingt-dix, les Somatiques ont largement pénétré le monde de la danse contemporaine française, et elles ont certainement contribué à deux changements importants. D'une part, une diversification des modèles de "compagnies" qui fait désormais cohabiter le modèle classique (directeur-chorégraphe, employeur d'interprètes-danseurs) avec d'autres (collectifs, "coalitions temporaires", rassemblements d'ordres divers). Cette diversification est corollaire au bouleversement des processus de travail: matériaux improvisés, créations collectives, composition instantanée, chorégraphies de l'imperceptible ${ }^{4} . .$. Le sentir et les processus d'invention du geste sont devenus objets chorégraphiques au même titre que la forme (ou le résultat) gestuel, qui prédominait jusque-là. Les "danses à sentir» cohabitent désormais avec les plus conventionnelles «danses à regarder».

Cette mise en lumière du sentir a transformé en profondeur non seulement la nature des catégories "chorégraphe/interprète», mais aussi la nature des rapports sociaux et politiques qui les soutiennent. Le deuxième changement - non moins politique - concerne l'espace social de la danse. Sortir la danse de ses studios et théâtres, l'étendre au-delà du seul spectacle et de sa préparation, travailler avec «tout le monde»; non-danseurs, mais aussi publics dits fragiles: enfants, personnes porteuses de handicaps, personnes hospitalisées... Les Somatiques donnent des outils que les danseurs peuvent partager avec les non-danseurs sans que s'engage d'emblée une hiérarchie entre virtuoses et amateurs, "valides » et "handicapés », experts et incompétents. Surtout, elles ont un matériau central qui semble le plus largement partagé, et semble à ce titre gage d'une par- faite démocratie: l'espace du sentir et de la perception, partagé tant par les danseurs virtuoses que par des enfants autistes, des adultes poly-handicapés, des seniors en fauteuil, etc.

Ce troisième cercle de douceurs serait donc politique: confrontées à certains contextes sociaux - celui du monde de la danse contemporaine, et ceux des publics dits fragilisés - les Somatiques offrent des outils pour redistribuer le «pouvoir d'agir» à partir de divers "savoir sentir»; des qualités d'écoute, une structuration du temps, qui s'opposent radicalement aux normes dominantes (tout particulièrement dans le champ médical et social). Leurs douceurs intensives et attentionnelles ouvriraient vers des modes de présence et d'écoute propices à défaire les hiérarchies relationnelles, et à rouvrir un pouvoir d'agir que la fixation des rôles (interprète, patient, professeur, employeur, usager social, etc.) empêchait. Enfin, les Somatiques seraient «par nature», et particulièrement du fait de leur structuration pédagogique, rétives à l'imposition de normes.

\section{VIOLENCES SOMATIQUES}

C'est sur cette question délicate des normes que je voudrais maintenant m'arrêter: non pas sur les débats internes sur l'existence ou non d'un «modèle » ou d'un "idéal» au sein de certaines méthodes, mais sur les usages normatifs qui peuvent être faits des douceurs somatiques. En effet, parmi les discours issus du monde des Somatiques, l'idée que celles-ci seraient «intrinsèquement » subversives (le sentiment de marginalité se substituant, me semble-t-il, à la notion de subversion) circule assez largement, alors même que l'engagement politique dans ses formes traditionnelles est rare parmi les praticiens somatiques.

Pourtant, c'est dans leurs usages qu'on peut interroger la fonction critique, ou subversive, des Somatiques; sans ignorer qu'elles peuvent parfois se laisser assujettir à l'ordre même qu'elles prétendent critiquer. Un premier exemple est celui qui fait des basses intensités non pas un moyen, mais un objectif. Ces basses intensités, en effet, sont un moyen d'explorer des perceptions affinées; lorsqu'elles se font passer pour la norme à atteindre, elles font des Somatiques une morale, s'autorisent à produire des jugements sur l'ensemble des pratiques gestuelles du monde social et, au-delà, prétendent détenir une vérité politique et sociale générale. On peut trouver des exemples de telles dérives dans certains ouvrages de Richard Shusterman ${ }^{5}$, qui porte des jugements sans appel sur diverses pratiques corporelles, sportives, sexuelles, politiques... dont les qualités comportent grandes amplitudes, vitesse, dynamiques explosives, sensations extrêmes.

On peut interpréter ces dérives comme une confusion entre "la fin et les moyens» et une mauvaise compréhension des Somatiques; mais dans nombre d'autres situations, les basses intensités, le tonus paisible, la convention de silence et la réduction du mouvement à des amplitudes minimales viennent soutenir les forces disciplinaires. L'introduction des pratiques somatiques à l'école, dans le travail social ou socio-éducatif, voire en milieu carcéral, se fait parfois sur un malentendu: si le praticien est convaincu d'œuvrer pour «le mieux-être» des usagers, il ne tardera pas à devoir choisir sa position quant aux attentes des professionnels avec lesquels > 
NOTES

6. Agnès Dufour (voir p. 27)

7. François Jullien,

Éloge de la Fadeur, éd.

P. Picquier, 1991, p. 19

8. Moshe Feldenkrais

(1904-1984) était

physicien et judoka.

La méthode qu'il

a développée au

long des années

40 à 80 revendique

explicitement ces deux

sources - et bien d'autres.

9. Voir p. 28.

LÉGENDES

- Page précédente et

ci-dessus à gauche

Une lente mastication,

chorégraphie de

Myriam Gourfink

interprétée par

Clément Aubert,

Clémence Coconnier,

Céline Debyser, Carole

Garriga, Kevin Jean,

Deborah Lary, Julie

Salgues, Françoise

Rognerud, Nina Santes,

Véronique Weil.

Photographies:

Laurent Paillier /

photosdedanse.com

- Ci-dessus au centre:

Sensationnelle,

dispositif chorégra-

phique de Julie Nioche

et Isabelle Ginot

(A.I.M.E.), pour un

danseur, un spectateur

et un "toucheur".

Festival C'est comme

ça! 2013. Photographie:

Véronique Baudoux.

- Ci-dessus à droite

Stage de Body-Mind

Centering animé par

Bonnie Bainbridge

Cohen, en 2011, à

Zürich. Photographie:

Thomas Greil.
> il collaborera, et découvrira parfois que son travail est mis au service d'un projet politique et social contradictoire avec le projet somatique. Faut-il «aider des détenus à garder leur calme»? Les enfants qui ne peuvent rester immobiles sur une chaise doivent-ils apprendre à le faire? Les précaires suivis par les services sociaux en vue d'une "réinsertion dans le monde du travail» désirent-ils vraiment améliorer leur "posture » et leur "présentation personnelle», ou ont-ils seulement incorporé les injonctions de ceux qui ont la main sur leurs droits sociaux? L'érudition de la sensibilité sert-elle autre chose pour les danseurs qu'une soumission à une exigence de virtuosité et de rendement toujours plus grande, et pas toujours choisie?

\section{FADEURS, NEUTRES, CENTRES... ÉTRANGETÉS}

C'est sans doute à condition qu'elle demeure pensée dans ses liens avec d'autres dynamiques, d'autres intensités, que la douceur des Somatiques peut demeurer douce. Il suffit d'écouter les danseurs qui en parlent: s'il s'agit de ralentir, c'est pour mieux pouvoir retourner vers la vitesse; s'il s'agit de s'écouter, c'est pour mieux pouvoir se maintenir, sans les subir, au sein de certaines violences - violences de la vitesse, des impacts physiques, et parfois de l'imaginaire. «Le neutre de Feldenkrais me permet non de cesser d'être en colère, mais au contraire de pouvoir aller vers la colère - tout en étant capable de revenir à un neutre avant de rentrer chez $\mathrm{moi}^{6}$ ! 》 Dans ces discours, l'usage des Somatiques est tendu vers un «ailleurs» du geste: on revient aux Somatiques pour pouvoir continuer à danser, pour passer d'un style chorégraphique à l'autre, pour renouveler son propre imaginaire. C'est pourquoi elles sont souvent associées à la notion de neutre: non pas comme idéal ou absolu (comme par exemple les prétendus «placements neutres » censés permettre toutes les danses), mais plus proche de ce que François Jullien définit dans la pensée chinoise comme la «fadeur»: "Quand les diverses saveurs, cessant de s'opposer les unes aux autres, restent contenues dans la plénitude: le mérite de la fadeur est de nous faire accéder à ce fond indifférencié des choses; sa neutralité exprime la capacité inhérente au centre 7 . » Très présente dans les arts martiaux orientaux, une telle notion du neutre comme "centre » sous-tend les logiques de nombreuses Somatiques, et particulièrement Feldenkrais ${ }^{8}$. Le neutre, ici, se comprend comme le point (postural, mental, gravitaire...) à partir duquel toute direction est possible. Autrement dit, il n'est pas une posture figée, «parfaitement verticale» ou centrale, mais il est neutre parce que, loin de les exclure, il contient la possibilité de tous les extrêmes. On peut comprendre la logique «martiale» de cette notion: le combattant doit être "prêt à tout», et toute préférence ou inclination - mentale, posturale, dynamique - est une faille que saura utiliser son adversaire. Aussi, le neutre n'est pas une forme prédéfinie à rechercher et maintenir, il est un point d'équilibre qui ne cesse de varier en fonction des forces en présence. Et il est surtout recherche de changement et de transformation plutôt que de fixation.

Pour les danseurs, les Somatiques ouvrent un passage vers un neutre qui permet et contient la possibilité de tous les excès: la vitesse, la virulence, la colère, l'explosion, la force extrême, la violence... ou encore le calme, l'immobilité... Les Somatiques ne contiennent pas toutes les danses, et ne constituent pas le «neutre» de toutes les danses. Mais elles constituent un point d'appui qui permet au danseur de maintenir un écart avec les danses qu'il est amené à traverser. "Se préparer à être étranger $^{9}$ ", selon la belle formule de Jean-Christophe Paré, ce serait s'assurer d'une hétérogénéité du geste, maintenir d'autres corporéités à travers celles que construit une danse donnée, ou en alternance avec elles. Les Somatiques sont douces, parce qu'elles forment un espace d'étrangeté à la danse, et une alternative aux forces dominantes: celles des formes chorégraphiques majoritaires, celles des pratiques de soin, ou encore celles des pédagogies du modèle. Elles sont douces parce qu'elles constituent, au sein de paysages normatifs fortement 
coercitifs, des espaces de retrait où leurs usagers (danseurs, patients, élèves, personnes handicapées...) peuvent élaborer des choix, se soustraire au jugement, élaborer des échelles de valeurs singulières. Autrement dit, elles sont douces parce qu'elles autorisent de nouveaux pouvoirs d'agir, et leur douceur est indissociable de ce à quoi elles s'opposent.

\section{Comment la danse pénètre-t-elle les Somatiques?}

Aussi, si les Somatiques forment la douceur de la danse parce qu'elles lui sont étrangères, quel serait l'étranger des Somatiques, qui formerait leur douce étrangeté? Lorsqu'on évoque les rapports entre Somatiques et danse, c'est toujours dans le sens des bénéfices et des transformations que les premières apportent à la seconde (gains techniques et sanitaires, parfois influences techniques et pédagogiques, plus rarement influence sur les processus de travail et de création). Alors qu'aujourd'hui un nombre de plus en plus grand de danseurs sont également praticiens somatiques, comment la danse pénètret-elle les Somatiques, et que leur fait-elle? En naviguant entre normes somatiques et normes chorégraphiques, entre pratiques "entre danseurs professionnels" et auprès de publics divers, les danseurs ont une place privilégiée pour éprouver les limites d'une pratique qui se fige. Ils ont aussi une palette d'outils et d'expériences trop riche pour se laisser enfermer dans une grammaire (les «principes » d'une méthode ou d'une autre) lorsque celle-ci devient une fin en soi. Lorsque ses "principes» de douceur heurtent - parfois avec violence - le public à qui ils s'adressent. Comment enseigner Feldenkrais aux enfants - qui n'aiment guère la lenteur? Comment ajuster la règle du "minimum d'effort » à des contextes où le relâchement peut être dangereux, comme dans la voltige? Comment les Somatiques sont-elles remises en cause lorsqu'elles rencontrent des contextes disciplinaires (comme certaines écoles de danse) et cependant désirables, et désirés par les danseurs? C'est ce que bien des danseurs intervenant dans ce numéro de Repères, cahier de danse nous enseignent: Sophie Lessard, qui façonne avec ses élèves des séances individuelles inspirées à la fois de plusieurs méthodes somatiques et de ses recherches de danseuse; Agnès Dufour, qui transporte et réinvente sa pratique Feldenkrais auprès d'enfants ou d'adultes porteurs de handicap; Régine Chopinot, attentive à ne pas faire du yoga une "méthode»; Noëlle Simonet, qui invente avec la cinétographie Laban une nouvelle modalité de dialogue empathique...

Ce sera donc ma conclusion en forme d'invitation aux danseurs: introduire de l'étranger au sein des pratiques chaque fois qu'elles se ferment sur leurs questions identitaires. Observer ce que ces règles définitionnelles d'une méthode produisent de singulier (ce qui fait que Feldenkrais est Feldenkrais) et ce qu'elles excluent de leur univers gestuel. Introduire le délire dans la syntaxe parfois sévère de ces méthodes. Se rappeler que la douceur ne leur est pas intrinsèque, qu'elle est toujours relative et relationnelle. Et ne jamais perdre de vue qu'elles reposent toutes sur une utopie de la liberté. 